\title{
ПСИХОСОМАТИКА
}

УДК 616.89-008.454:159.953.6:616.89-008-47:159.944:616.12-008.331.1:616.61:159.9.072.4

Для цитирования: Воронцов С.А., Хритинин Д.Ф., Макарова И.Ю. Риск когнитивных нарушений у больных артериальной гипертензией с факторами риска хронической болезни почек. Сибирский вестник психиатрии и наркологии. 2018; 4 (101): 93-97. https://doi.org/10.26617/1810-3111-2018-4(101)-93-97

\section{Риск когнитивных нарушений у больных артериальной гипертензией с факторами риска хронической болезни почек}

Воронцов С.А. ${ }^{1}$, Хритинин Д.Ф. ${ }^{2}$, Макарова И.ю. ${ }^{1}$

${ }^{1}$ Объединенный санаторий «Подмосковье» УДП РФ

142072, Московская обл., г. Домодедово, тер. Санатория «Подмосковье», стр. 25

${ }^{2}$ Первый Московский государственный медичинский университет имени И.М. Сеченова 119991, Москва, ул. Трубеикая, д. 8, стр. 2

\section{PEЗЮME}

Цель работы: оценить риск возникновения когнитивных нарушений у больных артериальной гипертензией с наличием факторов риска и без факторов риска хронической болезни почек. Материалы и методы. Обследовано 200 пациентов с диагнозом артериальная гипертензия I-II степени и наличием/отсутствием факторов риска хронической болезни

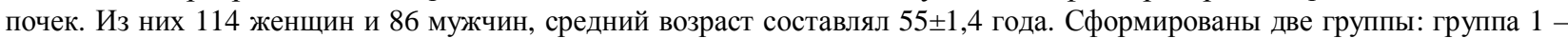
104 пациента, группа 2 - 96 пациентов. Исследование проводилось с использованием проб 10 слов, 5 фигур, отсчитывание, таблицы Шульте, шкал MMSE и FAB. Результаты. Выявлено, что у пациентов с артериальной гипертензией и факторами риска хронической болезни почек по сравнению с пациентами с артериальной гипертензией без факторов риска поражения почек ниже показатели слухоречевой памяти, концентрации произвольного внимания, скорости умственной работоспособности, а риск возникновения и развития когнитивных нарушений в 1,47 раза выше.

\section{Ключевые слова: артериальная гипертензия, хроническая болезнь почек, когнитивные нарушения.}

\section{ВВЕДЕНИЕ}

Артериальная гипертензия (АГ) является широко распространенным сердечно-сосудистым заболеванием (СС3), которое встречается более чем у $40 \%$ взрослого населения России [1]. Играя важную роль в развитии инфаркта миокарда, почечной недостаточности и мозгового инсульта, АГ признана одним из важных и независимых факторов риска развития когнитивных нарушений [2, 3]. При АГ нарушения затрагивают практически все виды когнитивных процессов: ухудшение внимания, снижение памяти, замедление темпа скорости реакции $[4,5]$. АГ ведет к изменениям в структуре головного мозга, что негативно сказывается на состоянии когнитивных процессов $[6,7,8]$. По результатам ряда современных исследований показано, что независимым фактором риска когнитивных нарушений $(\mathrm{KH})$ может являться хроническая болезнь почек (ХБП) [9, 10, 11], которая намного чаще диагностируется у пациентов с СС3 $[12,13,14]$ и увеличивает степень когнитивных нарушений пропорционально степени снижения почечных функций [15]. Таким образом, проблема когнитивных нарушений при АГ и ХБП по-прежнему является актуальной и требует дальнейшей научной разработки для создания комплексных программ ведения этой категории пациентов.

\section{ЦЕЛЬ ИССЛЕДОВАНИЯ}

Оценить риск возникновения когнитивных нарушений у пациентов с АГ и факторами риска ХБП по сравнению с пациентами с АГ без факторов риска почечных нарушений.

\section{МАТЕРИАЛЫ И МЕТОДЫ}

Было обследовано 200 пациентов ФГБУ Объединенный санаторий «Подмосковье» УДП РФ с диагнозом артериальная гипертензия I-II степени (средняя продолжительность заболевания составляла 15,6 \pm 2.4 года). Среди них было 114 (57\%) жен-

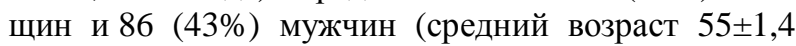
года). Пациенты были разделены на две группы. В группу 1 вошли 104 пациента с АГ и факторами риска ХБП, в группу 2 - 96 пациентов с АГ без факторов риска ХБП.

За факторы риска ХБП, согласно Клиническим Практическим Рекомендациям KDIGO [16] и Научного Общества Нефрологов России [17], было принято снижение скорости клубочковой фильтрации (СКФ) от 60 до 90 мл/мин/1,73 м² и повышение уровня альбуминурии (АУ) от 10 до 30 мг/л. В анамнезе у пациентов группы 1 ранее не было указаний на наличие почечной патологии, что позволило считать обнаружение факторов риска ХБП впервые выявленным. В исследование не включали пациентов с сахарным диабетом, в стадии декомпенсации сопутствующих заболеваний, при наличии в анамнезе инфаркта миокарда давностью менее 5 лет. Диагноз АГ у пациентов был установлен на досанаторном этапе в результате обследований в стационарных условиях. Для оценки наличия факторов риска ХБП у всех пациентов определялись концентрация альбумина в моче и уровень сывороточного креатинина, на основании которого по формуле CKD-EPI CR рассчитывали СКФ. 
Для оценки когнитивных нарушений применяли методики 10 слов А.Р. Лурия (слухоречевая память), 5 фигур (зрительная память), таблицы Шульте (концентрация и объем произвольного внимания), отсчитывание (скорость умственной работоспособности). Также применялась краткая шкала комплексной оценки когнитивных функций (Mini-Mental State Examination, MMSE) и батарея тестов на лобную дисфункцию (Frontal Assessment Battery, FAB).

Статистическую обработку материала проводили с использованием пакета программ SPSS Statistics 18.00. Статистическая значимость частотных различий проверялась критерием $\chi^{2}$ Пирсона. Различия считали достоверными при значении $\mathrm{p}<0,05$. Для оценки вероятности проявления когнитивных нарушений у пациентов с АГ в зависимости от факторов риска использовался показатель относительного риска (relative risk, RR) с расчетом границы 95\% доверительного интервала.

\section{РЕЗУЛЬТАТЫ И ОБСУЖДЕНИЕ}

У пациентов группы 1 с АГ и факторами риска ХБП по сравнению с пациентами группы 2 с АГ без факторов риска ХБП отмечалось более выраженное нарушение когнитивных процессов. Средние показатели когнитивных процессов в группе 1 были до- стоверно ниже, чем в группе 2. Индивидуальные показатели у пациентов с АГ и факторами риска ХБП чаще отражали картину умеренных когнитивных нарушений, чем у пациентов с АГ без факторов риска ХБП. В группе 1 частота выявления умеренных когнитивных расстройств составила $64,4 \%$, в группе $2-47,9 \%(\mathrm{p}<0,05)$.

В обеих группах показатели произвольного внимания были снижены, наблюдалась повышенная утомляемость пациентов, однако уровень концентрации произвольного внимания в группе 1 был достоверно хуже $(75,1 \pm 5,7$ сек. и $71,4 \pm 6,2$ сек. соответственно, $\mathrm{p}<0,05)$. Скорость умственной работоспособности в группе 2 была несколько ниже нормы, тогда как в группе 1 этот показатель был существенно снижен $(81,8 \pm 5,9$ сек. в группе 1 против $67,7 \pm 4,5$ сек. в группе $2, \mathrm{p}<0,05)$ (рис. 1 ).

Это свидетельствует о наличии у пациентов с АГ, особенно с факторами риска ХБП, нейродинамических нарушений по типу утомляемости, снижения умственной работоспособности, флуктуаций произвольного внимания. Причем у пациентов с АГ и факторами риска ХБП нейродинамические нарушения оказались достоверно более выраженными, чем у пациентов с АГ без факторов риска ХБП.

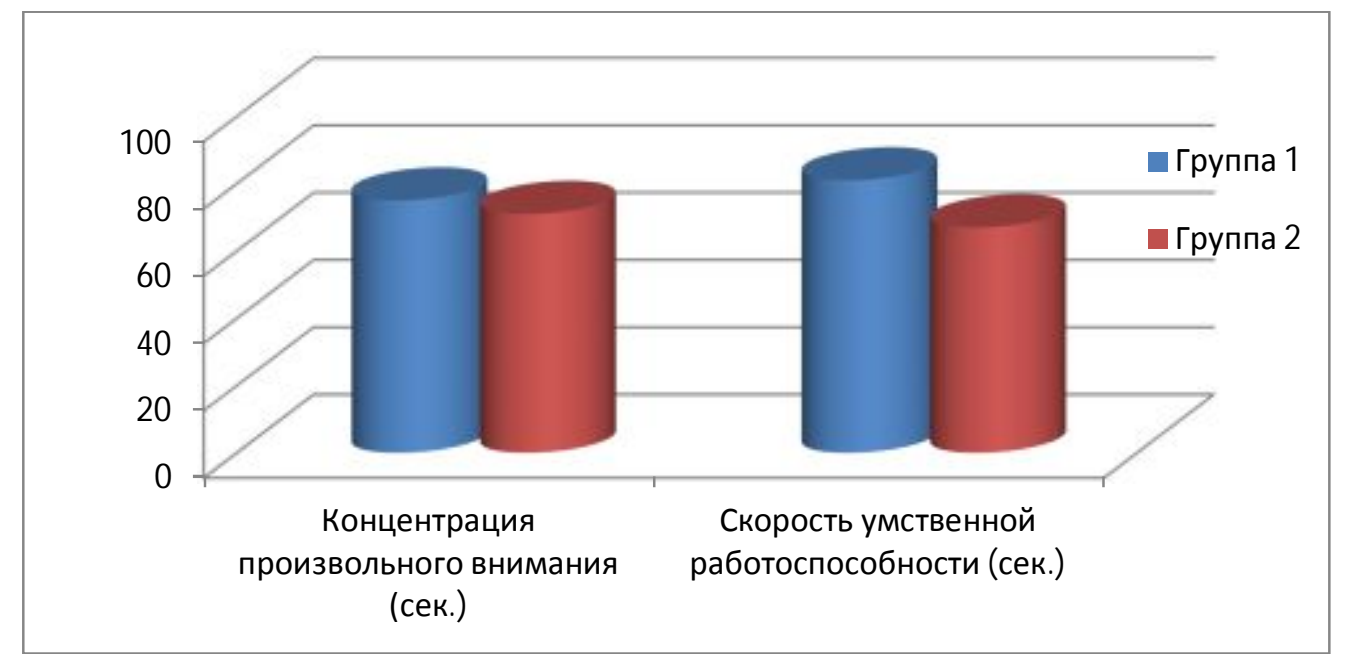

\section{Р и с у н о к 1 . Показатели внимания и скорости умственной работоспособности в группе 1 и группе 2}

Более низкий объем слухоречевой памяти отмечался в группе $1(6,3 \pm 1,4$ балла, p<0,01), средний балл данного показателя в группе 2 входил в норма-

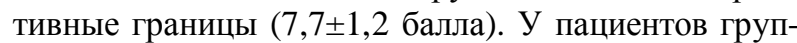
пы 1 по сравнению с пациентами группы 2 отмечалась худшая фиксация слухоречевых стимулов в условиях гетерогенной интерференции $(4,5 \pm 0,8$

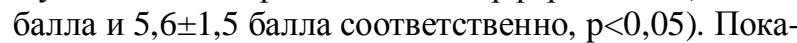
затели зрительной памяти в обеих группах соответствовали нормативным значениям (рис. 2).

Это указывает на снижение качества краткосрочной фиксации слухоречевых стимулов с дальнейшим затруднением долгосрочного запоминания, особенно в условиях гетерогенной интерференции, при относительной сохранности зрительной памяти.
Средний балл шкалы MMSE у пациентов группы 1 составил 24,8 $\pm 1,2$, что отражает клиническую картину легких когнитивных нарушений. У пациентов

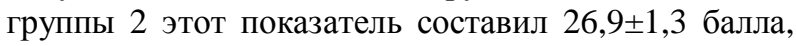
что приближено к границе нормы. При оценке индивидуальных показателей у $31,3 \%$ пациентов с АГ и факторами риска ХБП был обнаружен уровень умеренных когнитивных расстройств, среди пациентов с АГ без факторов риска ХБП умеренные когнитивные расстройства отмечались у достоверно меньшего количества больных $(17,9 \%)$. Результаты шкалы FАВ указывали на достаточную сохранность лобных долей мозга и подкорковых образований у пациентов обеих групп (16,0 $\pm 0,3$ балла и $16,7 \pm 0,8$ балла соответственно, $\mathrm{p}<0,05)$. 


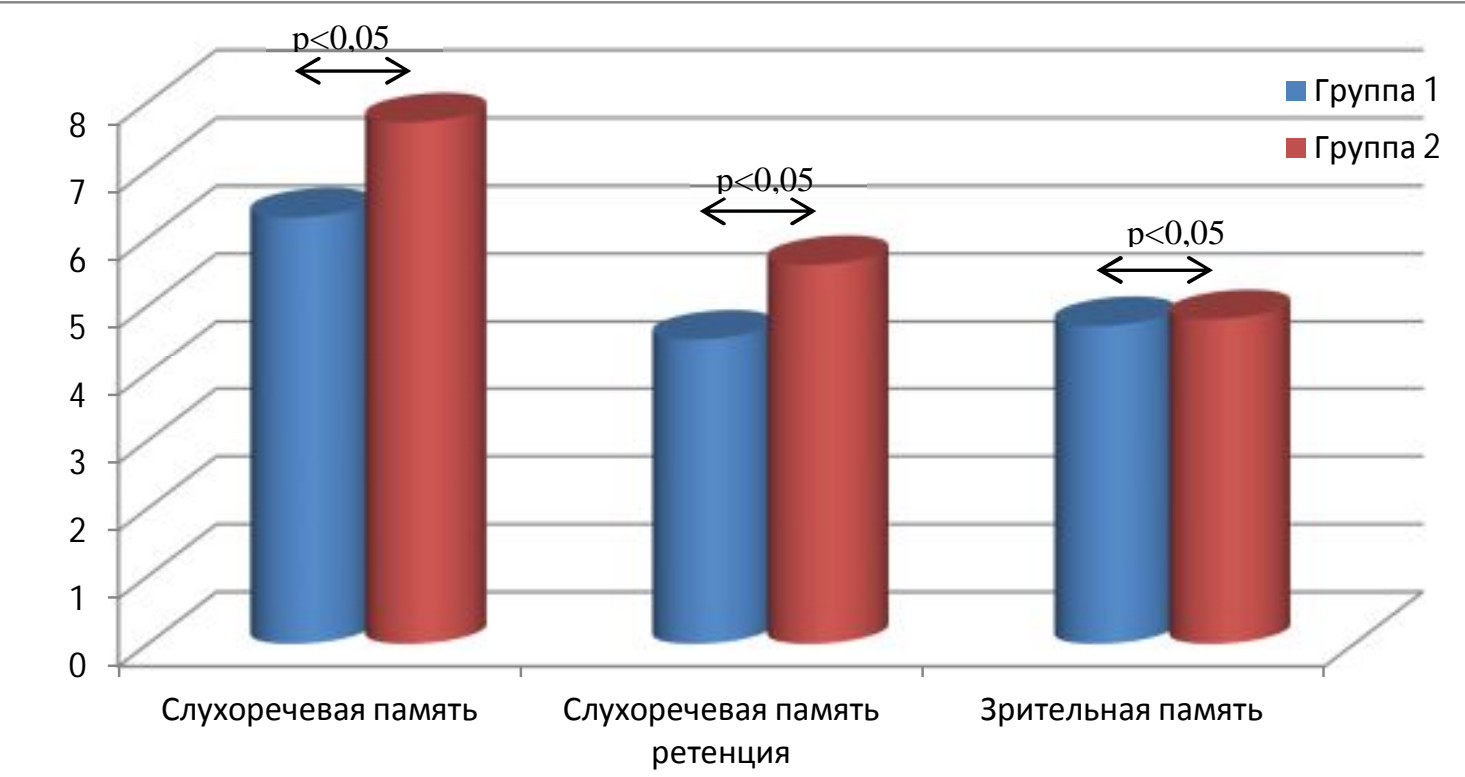

Р и с у н о к 2. Показатели слухоречевой и зрительной памяти в группе 1 и группе 2

Был определен относительный риска возникновения когнитивных нарушений у пациентов с АГ в зависимости от наличия или отсутствия факторов риска ХБП. На основании расчета относительного риска с 95\% доверительным интервалом (RR 1,47; 95 ДИ 1,160-1,864) у пациентов с интегрированным риском по АГ и ХБП риск появления КН в 1,47 раза выше, чем у пациентов только с АГ.

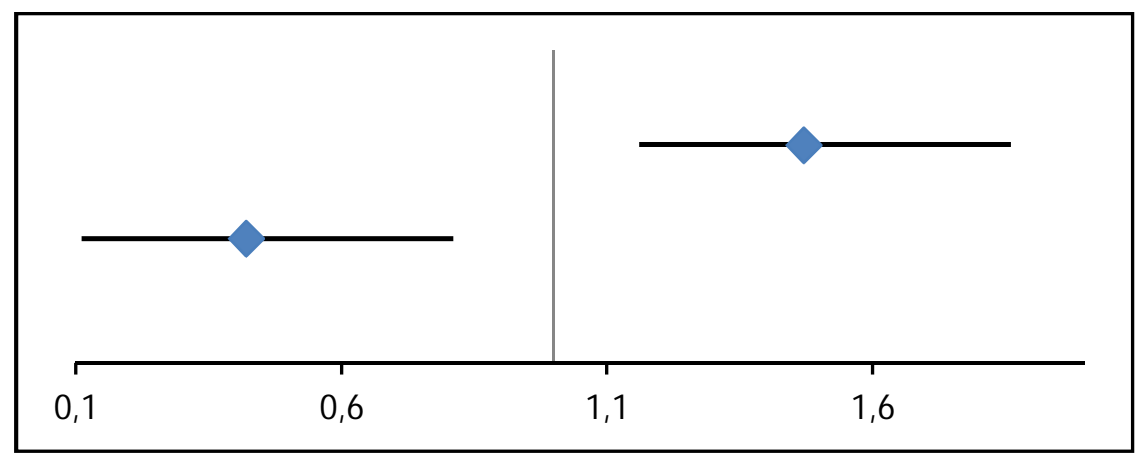

Группа 1

Группа 2

\section{Р и с у н о к 3. Относительный риск КН в группе 1 и группе 2}

Таким образом, в ходе исследования установлено, что у подавляющего большинства обследованных пациентов с АГ отмечаются когнитивные нарушения различной степени выраженности и спектра (расстройства внимания, мнестические расстройства, ухудшение динамики умственной работоспособности).

У пациентов с АГ I-II степени и факторами риска ХБП по сравнению с пациентами только с АГ выявлены достоверно более низкие показатели слухоречевой памяти и нейродинамического компонента (как объема и концентрации произвольного внимания, так и скорости умственной работоспособности) при относительной сохранности на данной стадии развития заболевания зрительной памяти и функции лобных долей головного мозга. Присоединение к артериальной гипертензии хронической болезни почек еще на стадии факторов риска в 1,47 раза повышает риск развития когнитивных наруше- ний, что указывает на важность ранней диагностики факторов риска ХБП.

\section{ЗАКЛЮЧЕНИЕ}

Оценка риска вероятности возникновения когнитивных нарушений у больных артериальной гипертензией с негативным воздействием факторов риска хронической болезни почек позволит своевременно начать лечебные мероприятия по предотвращению развития более выраженных стадий ХБП, что, в свою очередь, будет являться профилактикой возникновения и дальнейшего развития когнитивных нарушений.

\section{КОНФЛИКТ ИНТЕРЕСОВ}

Авторы декларируют отсутствие явных и потенциальных конфликтов интересов в связи с публикацией данной статьи.

\section{ИСТОЧНИК ФИНАНСИРОВАНИЯ}

Авторы заявляют об отсутствии финансирования при проведении данного исследования. 


\section{СООТВЕТСТВИЕ ПРИНЦИПАМ ЭТИКИ}

Исследование когнитивных нарушений у больных артериальной гипертензией с наличием факторов риска и без факторов риска хронической болезни почек проведено с соблюдением норм современной биомедицинской этики и этических стандартов, разработанных в соответствии с Хельсинской декларацией ВMА.

\section{ЛИТЕРАТУРА}

1. Ощепкова Е. В., Чазова И.Е. Итоги реализации Федеральной целевой программы по профилактике и лечению артериальной гипертензии в России в 2002-2012 гг. Вестник РАMH. 2013; 68 (2): 4-11.

2. Knopman D.S. Cerebrovascular disease and dementia. British J. Radiol. 2007; 80: 121-127.

3. Singh-Manoux A., Marmot M. High blood pressure was associated with cognitive function in middle-age in the Whitehall II study. J. Clin. Epidemiol. 2005; 58: 1308-1315.

4. Суханов А.В., Денисова Д.В. Ассоциация артериального давления, пульса и когнитивных функций в подростковом возрасте: популяционное исследование. Артериальная гипертензия. 2010; 16 (4): 378-384.

5. Peter L., Dome P., Rihmer Z. et al. Cardiovascular disorders and depression: a review of epidemiological and possible etiological data. Neuropsychopharmacol. 2008; 10 (2): 81-90.

6. Поляков В.М. Когнитивные нарушения при эссенциальной артериальной гипертензии (обзор литературы). Бюллетень Восточно-Сибирского научного центра Сибирского отделения Российской Академии медицинских наук. 2013. 22 (1): 180-184.

7. Elkins J.S., Yaffe K., Cauley J.A. et al. Pre-existing hypertension and the impact of stroke on cognitive function. Ann. Neurol. 2005; 58: 68-74.

8. Seliger S.L., Siscovick D.S., Stehman-Breen C.O., Gillen D.L., Fitzpatrick A., Bleyer A. et al. Moderate renal impairment and risk of dementia among older adults The Cardiovascular Health Cognition study. J Am Soc Nephrol. 2004; 15: 1904-1911.

9. Etgen T., Chonchol M., Förstl H., Sander D. Chronic kidney disease and cognitive impairment: a systematic review and metaanalysis. Am J Nephrol. 2012; 35(5): 474-482.

10. Helmer C., Stengel B., Metzger M., Froissart M., Massy Z.A., Tzourio C. et al. Chronic kidney disease, cognitive decline, and incident dementia: the 3C Study. Neurology. 2011; 77 (23): 2043-2051.

11. Yaffe K., Ackerson L., Kurella Tamura M. Le Blanc P., Kusek J.W., Sehgal A.R., Cohen D. et al. Chronic kidney disease and cognitive function in older adults: findings from the chronic renal insufficiency cohort cognitive study. J. Am Geriatr. Soc. 2010; 58 (2): 338-345.

12. Пигарева Ю.А., Авдошина С.В., Дмитрова Т.Б., Ефремовцева М.А., Тигай Ж.Г. Распространенность хронической болезни почек среди пациентов терапевтического стационара. Клиническая нефрология. 2012; 3: 4-9.

13. Серов В.А., Шутов А.М., Мензоров М.В., Макеева Е.Р., Серова Д.В. Эпидемиология хронической болезни почек у больных с хронической сердечной недостаточностью. Нефрология. 2010; 14 (1): 50-55.

14. Karalliedde J., Gnudi L. Endothelial factors and diabetic nephropathy. Diabetes Care. 2011; 34 (2): 291-296.

15. Raphael K.L., Wei G., Greene T., Baird B.C., Beddhu S. Cognitive function and the risk of death in chronic kidney disease. Am. J. Nephrol. 2012; 35(1): 49-57.

16. Практические рекомендации KDIGO по диагностике, профилактике и лечению минеральных и костных нарушений при хронической болезни почек (ХБП-МКН). Краткое изложение рекомендаций. Нефрология. 2011; 15 (1): 88-95.

17. Смирнов А.В., Шилов Е.М., Добронравов В.А., Каюков И.Г., Бобкова И.Н., Швецов М.Ю., Цыгин А.Н., Шутов А.М. Национальные рекомендации. Хроническая болезнь почек: основные принципы скрининга, диагностики, профилактики и подходы к лечению. Нефрология. 2012; 16 (1): 89-115.

Поступила в редакцию 8.05.2018 Утверждена к печати 6.11.2018

Воронцов Сергей Анатольевич, кандидат медицинских наук, директор ФГБУ «Объединенный санаторий «Подмосковье» Управления делами президента РФ, Россия, г. Домодедово.

Макарова Инна Юрьевна, клинический психолог ФГБУ «Объединенный санаторий «Подмосковье» Управления делами президента РФ, Россия, г. Домодедово

Хритинин Дмитрий Федорович, доктор медицинских наук, член-корреспондент РАН, профессор кафедры психиатрии и наркологии Первого государственного медицинского университета имени И.М. Сеченова.

Макарова Инна Юрьевна, innamakarova@mail.ru

УДК 616.89-008.454:159.953.6:616.89-008-47:159.944:616.12-008.331.1:616.61:159.9.072.4

For citation: Vorontsov S.A., Khritinin D.F., Makarova I.Yu. Risk of cognitive impairment in hypertensive patients with risk factors for chronic kidney disease. Siberian Herald of Psychiatry and Addiction Psychiatry. 2018; 4 (101): 93-97. https://doi.org/10.26617/1810-3111-2018-4(101)-93-97

\title{
Risk of cognitive impairment in hypertensive patients with risk factors for chronic kidney disease
}

\author{
Vorontsov S.A. ${ }^{1}$, Khritinin D.F. ${ }^{2}$, Makarova I.Yu. ${ }^{1}$ \\ ${ }^{1}$ United Sanatorium "Moscow region” of Office of Presidential Affairs of the Russian Federation \\ Territory of the Sanatorium "Moscow region", building 25, 142072, Moscow Region, Domodedovo, Russian Federation \\ ${ }^{2}$ I.M. Sechenov First Moscow State Medical University \\ Trubetskaya Street 8, building 2, 119991, Moscow, Russian Federation
}

\section{ABSTRACT}

Objective: to assess the risk of cognitive impairment in patients with hypertension in the presence of risk factors and without risk factors for chronic kidney disease. Materials and Methods. 200 patients with the diagnosis of arterial hypertension of the first and second degree and presence/absence of risk factors of chronic kidney disease were examined. Of those, 114 were women and 86 were men, with mean age of $55 \pm 1.4$ years. Two groups were formed: group $1-104$ patients, group $2-96$ patients. The study 
was conducted using psychological tests (10 words, 5 figures, counting, Schulte table) and clinical psychological scales MMSE and FAB. Results. It was found that in patients with hypertension and risk factors for chronic kidney disease compared with patients with hypertension without risk factors for kidney damage, the indices of auditory memory, the concentration of arbitrary attention, the speed of mental performance, and the risk of occurrence and development of cognitive disorders are 1.47 times higher.

\section{Keywords: arterial hypertension, chronic kidney disease, cognitive impairment.}

\section{REFERENCES}

1. Chazova I.E., Oshchepkova E.V. Itogi realizacii Federal'noj celevoj programmy po profilaktike i lecheniyu arterial'noj gipertenzii v Rossii v 2002-2012 godah [Results of the implementation of the Federal target program for the prevention and treatment of hypertension in Russia in 2002-2012]. Vestnik RAMN - Bulletin of the Russian Academy of Medical Sciences. 2013; 68 (2): 4-11 (in Russian).

2. Knopman D.S. Cerebrovascular disease and dementia. British J. Radiol. 2007; 80: 121-127.

3. Singh-Manoux A., Marmot M. High blood pressure was associated with cognitive function in middle-age in the Whitehall II study. J. Clin. Epidemiol. 2005; 58: 1308-1315.

4. Sukhanov A.V., Denisova D.V. Associaciya arterial'nogo davleniya, pul'sa i kognitivnyh funkcij v podrostkovom vozraste: populyacionnoe issledovanie [Associations of blood pressure, heart rate and cognitive function in the adolescents: A population-based study]. Arterial'naya gipertenziya - Arterial Hypertension. 2010; 16 (4): 378-384 (in Russian).

5. Peter L., Dome P., Rihmer Z. et al. Cardiovascular disorders and depression: a review of epidemiological and possible etiological data. Neuropsychopharmacol. 2008; 10 (2): 81-90.

6. Polyakov V.M. Kognitivnye narusheniya pri ehssencial'noj arterial'noj gipertenzii (obzor literatury) [Cognitive impairment in essential hypertension (literature review)]. Byulleten' VSNC SO RAMN - Bulletin of the East Siberian Scientific Center of the Siberian Branch of the Russian Academy of Medical Sciences. 2013; 22 (1): 180-184 (in Russian).

7. Elkins J.S., Yaffe K., Cauley J.A. et al. Pre-existing hypertension and the impact of stroke on cognitive function. Ann. Neurol. 2005; 58: 68-74.

8. Seliger S.L., Siscovick D.S., Stehman-Breen C.O., Gillen D.L., Fitzpatrick A., Bleyer A. et al. Moderate renal impairment and risk of dementia among older adults The Cardiovascular Health Cognition study. J Am Soc Nephrol. 2004; 15: 1904-1911.

9. Etgen T., Chonchol M., Förstl H., Sander D. Chronic kidney disease and cognitive impairment: a systematic review and metaanalysis. Am J Nephrol. 2012; 35(5): 474-482.

10. Helmer C., Stengel B., Metzger M., Froissart M., Massy Z.A., Tzourio C. et al. Chronic kidney disease, cognitive decline, and incident dementia: the 3C Study. Neurology. 2011; 77 (23): 2043-2051.
11. Yaffe K., Ackerson L., Kurella Tamura M. Le Blanc P., Kusek J.W., Sehgal A.R., Cohen D. et al. Chronic kidney disease and cognitive function in older adults: findings from the chronic renal insufficiency cohort cognitive study. J. Am Geriatr. Soc. 2010; 58 (2): 338-345.

12. Pigareva Yu.A, Avdoshina S.V, Dmitrova T.B., Yefremovtseva M.A., Tigay J.G. Rasprostranennost' hronicheskoj bolezni pochek sredi pacientov terapevticheskogo stacionara [Prevalence of chronic kidney disease in patients of clinic of internal diseases]. Klinicheskaya nefrologiya - Clinical Nephrology. 2012; 3: 4-9 (in Russian).

13. Serov V.A., Shutov A.M., Menzorov M.V., Makeeva E.A., Serov D.V. Ehpidemiologiya hronicheskoj bolezni pochek u bol'nyh s hronicheskoj serdechnoj nedostatochnost'yu [Epidemiology of chronic kidney disease patients with chronic heart failure]. Nefrologiya - Nephrology. 2010; 14 (1): 50-55 (in Russian).

14. Karalliedde J., Gnudi L. Endothelial factors and diabetic nephropathy. Diabetes Care. 2011; 34 (2): 291-296.

15. Raphael K.L., Wei G., Greene T., Baird B.C., Beddhu S. Cognitive function and the risk of death in chronic kidney disease. Am. J. Nephrol. 2012; 35(1): 49-57.

16. Prakticheskie rekomendacii KDIGO po diagnostike, profilaktike i lecheniyu mineral'nyh i kostnyh narushenij pri hronicheskoj bolezni pochek (HBP-MKN). Kratkoe izlo-zhenie rekomendacij [KDIGO practical recommendations for the diagnosis, prevention and treatment of mineral and bone disorders in chronic kidney disease (CKD-MCS). Summary of recommendations]. Nefrologiya - Nephrology. 2011. 15 (1): 88-95 (in Russian).

17. Smirnov A.V., Shilov E.M., Dobronravov V.A., Kayukov I.G., Bobkova I.N., Shvetsov M.Yu., Tsygin A.N., Shutov A.M. Nacional'nye rekomendacii. Hronicheskaya bolezn' pochek: osnovnye principy skrininga, diagnostiki, profilaktiki i podhody $\mathrm{k}$ lecheniyu [National guidelines. Chronic kidney disease: basic principles of screening, diagnosis, prevention and treatment approaches]. Nefrologiya-Nephrology. 2012; 16 (1): 89-115 (in Russian).

Received May 8.2018 Accepted November 6.2018

Vorontsov Sergey A., PhD, Director of the United Sanatorium "Moscow region” of the Presidential Administration, Domodedovo, Russian Federation.

Makarova Inna Yu., clinical psychologist, United Sanatorium "Moscow region” of the Presidential Administration, Domodedovo, Russian Federation.

Khritinin Dmitry F., MD, corresponding member of RAS, professor of the Department of Psychiatry and Narcology of the First State Medical University named after I.M. Sechenov, Moscow, Russian Federation

Makarova Inna Yu., innamakarova@mail.ru 